AperTO - Archivio Istituzionale Open Access dell'Università di Torino

\title{
Gut Microbiota, Immune System, and Bone
}

\section{This is the author's manuscript}

Original Citation:

Availability:

This version is available http://hdl.handle.net/2318/1649792

since 2017-10-18T09:43:11Z

Published version:

DOI:10.1007/s00223-017-0331-y

Terms of use:

Open Access

Anyone can freely access the full text of works made available as "Open Access". Works made available under a Creative Commons license can be used according to the terms and conditions of said license. Use of all other works requires consent of the right holder (author or publisher) if not exempted from copyright protection by the applicable law. 


\section{(3) \\ UNIVERSITÀ DEGLI STUDI DI TORINO}

This is an author version of the contribution published on:

Questa è la versione dell'autore dell'opera:

Gut Microbiota, Immune System and Bone.

D'Amelio P, Sassi F.

Calcif Tissue Int. 2017 Sep 30. doi: 10.1007/s00223-017-0331-y.

The definitive version is available at:

La versione definitiva è disponibile alla URL:

[https://link.springer.com/article/10.1007\%2Fs00223-017-0331-y] 
Gut Microbiota, Immune System and Bone

D'Amelio $\mathrm{P}^{1}$, Sassi $\mathrm{F}^{1}$

${ }^{1}$ Gerontology and Bone Metabolic Diseases Section

Department of Medical Science University of Torino- Italy

Corresponding author and reprint request:

D'AmelioPatrizia MD, $\mathrm{PhD}$

Department of Medical Science,

University of Torino

CorsoDogliotti 14, 10126 Torino, Italy.

Tel: +390116336704-Fax: +390116636033

E-mail: patrizia.damelio@unito.it.

Running title: Microbiota and Bone 


\begin{abstract}
The gut microbiota (GM) is the whole of commensal, symbiotic and pathogenic microorganisms living in our intestine. The GM-host interactions contribute to the maturation of the host immune system, modulating its systemic response. It is well documented that GM can interact with nonenteral cells as immune cells, dendritic cells and hepatocytes, producing molecules as short-chain fatty acids, indole derivatives, polyamines and secondary bile acid. The receptors for some of these molecules are expressed on immune cells, and modulate the differentiation of $\mathrm{T}$ effector and regulatory cells: this is the reason why dysbiosis is correlated with several autoimmune, metabolic and neurodegenerative diseases.

Due to the close interplay between immune and bone cells, GM has a central role in maintaining bone health and influences bone turnover and density. GM can improve bone health also increasing calcium absorption and modulating the production of gut serotonin, a molecule that interacts with bone cells and has been suggested to act as a bone mass regulator. Thus, GM manipulation by consumption of antibiotics, changes in dietary habits and the use of pre- and probiotics may affect bone health.

This review summarizes evidences on the influence of GM on immune system and on bone turnover and density and how GM manipulation may influence bone health.
\end{abstract}

Keywords: osteoporosis, gut microbiota, bone, immune system, probiotics, inflammation 


\section{Introduction}

The whole of the commensal, symbiotic and pathogenic microorganisms living in our intestine has been defined gut microbiota (GM), it is acquired at birth and derives almost entirely from the mother, it changes accordingly to environmental factors as diet, diseases and use of drugs. The GM comprises about 1200 bacterial species, the main phyla represented are: Bacteroidetes, Firmicutes, Actinobacteria, Proteobacteria and Verrucomicrobia [1].Some of the identified species and of the common bacterial phyla varies between individuals [2], low microbial diversity have been identified as a risk factor for different chronic diseases as intestinal inflammatory diseases, obesity and insulin resistance $[3,4,5,6]$. Arumugam and colleagues suggested that individuals can be clustered according to the prevalence of different GM phyla and introduced the concept of "enterotypes"; according to this definition humans can be stratified on the basis of their microbial patterns dominated by Bacteroides, Prevotella or by Ruminococcus [7].

In physiological condition GM relationship with host is complex and comprehends various forms of symbiotic relationship as parasitic, commensal and mutualistic, GM helps in food digestion, in fighting pathogens and, during the first years of post-natal life, contributes to the maturation of the host immune system; during the whole life GM interacts with the host and contributes to the modulation of gut and systemic immunity. Immune homeostasis disruption is the causal mechanism of several chronic non-communicable human diseases (NCDs) as allergy, asthma, some autoimmune, cardiovascular and metabolic diseases, and neurodegenerative disorders. These disorders are characterized by a low grade of inflammation. Although inflammation and the pathways to disease are multifactorial, the altered gut colonization patterns, associated with decreasing microbial diversity, are a central theme and are increasingly implicated in the physiologic, immunologic, and metabolic deregulation seen in many NCDs. Altered GM-host interaction has been indicated as a possible cause of immune deregulation and increased inflammation associated with several NCDs [8].

This review summarizes evidences on the influence of GM on immune system and on bone turnover and density and how GM manipulation may influence bone health.

\section{GM influences immune system}

The interaction between immune system and GM has a central role in the maturation of immune system during the early post-natal period [9] and a role in the modulation of immune system and response to self-antigens during the whole life $[9,10]$, thus it has been suggested that dysbiosis may play a role in the development of diseases characterized by immune deregulation such as allergies, autoimmune, and inflammatory disorders.

The role of GM in the development and maturation of host immune system in the early post-natal life has been demonstrated in germ free (GF) mice, i.d. animal raised in sterile cages that maintains sterile gut. The use of this experimental model have shown that the absence of GM negatively influences the formation of lymphoid organs, in particular GF mice have defective formation of the spleen and mesenteric lymph nodes, the intestinal Peyer's patch are smaller and displays a reduced number of CD4+T cells and reduced production of IgA [11-16]. Also isolated lymphoid follicle and 
cryptopatches are reduced in GF mice [17, 18]. As regards immune cells different GM phyla where associated with the development of different $\mathrm{T}$ helper (Th) phenotypes: in animal model of rheumatoid arthritis (RA) the disease is reduced in GF mice thanks to a reduction of Th 17 [19], arthritic phenotypes is restored when GF animals are colonized with segmented filamentous bacteria, which enhances the differentiation and function of Th17 cells. In RA patients a relationship between the disease and Prevotellaceae has been suggested, in particular Prevotellae copri has been associated with increased risk of RA [20, 21], whereas Prevotella histicola seems to inhibit the development of arthritis [22].Colonization of GF animals with Bacterioides fragilis restores a correct balance between Th1 and Th2 cells and redirect lymphoid organogenesis [14]. Resident bacteria as segmented filamentous bacterium and in particular some Clostridia-related species, have been associated to Th cells development and to Tregs cells induction [23, 24]. GM modulates immune system through the production of molecules with immunomodulatory and anti-inflammatory function that are capable to influence immune cells [25, 26]. In particular GM produces several metabolites from digested food, by modifying host products and by the novo synthesis, amongst these molecules short-chain fatty acids (SCFAs) are the most widely investigated in the regulation of inflammation and immune system. It has been demonstrated that SCFAs have anti-inflammatory effects on intestinal mucosa, thus protecting the bowel from the development of inflammatory bowel disease [27-29] (Fig. 1).

SCFAs signals to several non-enteral cell types through G-protein-coupled receptors also named free fatty acid receptors (FFAR) [30-32], one of these receptors GPR109A/HCA2, is activated in immune system by butyrate [33], the signal between GM and immune system is fundamental to regulate the homeostasis and to maintain the balance between immune tolerance to commensals bacteria and immunity to pathogens. The interaction of butyrate and GPR109A/HCA2 cooperates in the generation of immune tolerance and, in particular, mediates Tregs development [28, 29, 34,35]. Butyrate regulates gene expression by inhibiting histone deacetylases (HDAC) [36], in particular butyrate inhibits HDAC1 and HDAC3 [37]. Also propionate acts as a less potent HDAC inhibitor [38]. Recently it has been suggested that inhibition of HADAC may increase Tregs development and function, hence this could be one of the mechanism by which GM enhances Treg generation in the gut [39].It has also been suggested that, depending on the cytokines milieu, interaction between SCFA and FFAR influences T cells differentiation not only towards Tregs, but also towards effector T cells. Park and colleagues suggested that, in certain conditions, SCFAs may induce T helper differentiation into Th1 and Th17 thus increasing the host defenses against pathogens [40]. SCFAs as butyrate and propionate also modulates antigens presentation inhibiting the development of dendritic cells by HDAC inhibition [41-44] and by interaction with FFAR [34, 45].

Beyond SCFAs, GM produces other metabolites from digested food that have important immunomodulatory function as indole derivatives and polyamines, these metabolites derive from dietary tryptophan and arginine respectively and have an indirect immune function. Indoles derivatives favor the integrity of the enteral mucosa and the barrier defense towards pathogens by stimulating the production of anti-microbial peptides, mucins, and proliferation of intestinal goblet cells. Polyamines as putrescine, spermidine, and spermine fulfill important roles in gene expression and proliferation; enhance the development and maintenance of the intestinal mucosa and resident immune cells (Fig. 1). An immunomodulating role have also been postulated for other GM products as metabolized bile acids, however physiological role for this metabolites in health and disease is still an open question [46]. 
GF mice have imbalance in T helper cells: reduced Treg, absence of Th17 cells and altered ratio between Th1 and Th2 with increased Th2 response [26], in these animals gut colonization with Bacteroides fragilis induces the development of Th1 cells thanks to the production of polysaccharide A [14]. Polysaccharide A is a bacterial product that influences T cells fate thought its interaction with the toll like receptor 2 , interacting with $\mathrm{T}$ cells it favors immune tolerance by inhibiting Th17 differentiation and favoring Tregs activity [47]. Other bacteria, as segmented filamentous bacteria and Clostridium spp.,were shown to influence Th phenotype, the first stimulates Th17 immune response, through ATP or serum amyloid A production by innate immunity cells, whereas the latter promotes Treg cell response trough SCFAs production [23, 48] (Fig. 1).

A recent study by Kim and colleagues suggests that GM may affect also B cells antibody production through SCFAs inhibition of HDAC and modulation of gene expression [49] however further studies are needed to clarify the underling mechanism.

Taken together these evidences suggest that GM influences T cells differentiation through the production of bacterial metabolites as SCFAs and polysaccharide A at least at the intestinal mucosa level, and T cells differentiation trough cognate bacterial antigens [50] (Fig. 1).

The majority of the evidences thus suggested that GM metabolites and antigens may influence immune regulation and hence dysbiosis may be the environmental factor responsible for some immune and inflammatory disorders, both at gut level as inflammatory bowel disease [51] and outside the gut as Rheumatoid Arthritis [52], type1 diabetes [53] and asthma [54]. However organs distant from gut, skin and lung are not in direct contact with GM, this implies that GM has the ability to communicate to the host immune system in distant organs as well as in the gut. These signals have been identified in GM derived products as lipopolysaccharide, SCFAs, and bile acid but also circulating antibodies or immune cells [2].

\section{Relationship between GM, immune system activation and bone loss}

Osteoporosis increases dramatically the risk of fractures: major osteoporotic fractures are a social and economic burden, in developed countries, the lifetime risk for osteoporotic fractures at the wrist, hip or spine is $30 \%$ to $40 \%$, very close to that for coronary heart disease. The number of new fractures in 2010 in the EU was estimated at 3,5 million, comprising approximately 620,000 hip fractures, 520,000 vertebral fractures, 560,000 forearm fractures and 1,800,000 other fractures [55]. Osteoporotic fractures impair patients' quality of life and increase mortality: $20 \%$ of elderly patients suffering from femoral fractures will die within a year, and $50 \%$ of the survivors will lose independence. The most frequent cause of bone loss is post-menopausal osteoporosis (PMO) that is driven by estrogen deficiency at menopause. In PMO there is an imbalance in bone turnover with increased bone resorption and reduced bone formation. It has been demonstrated both in experimental models and in humans that estrogen deficiency affects bone cells number and activation and bone turnover partially through their effect on immune system [56].During estrogen deficiency $\mathrm{T}$ cells increase their production of pro-inflammatory and pro-osteoclastogenic cytokines, such as TNF alpha and RANKL [57], however the reasons of this increased activity in osteoporotic women and not in non-osteoporotic subject is unknown, GM may be involved in the mechanism of PMO. 
Some papers suggest that the absence of GM influences bone mass, the majority of the findings demonstrate that GF mice have increased bone mass, whereas a single study by Schwarzer and colleagues [58] demonstrated that GF mice have a growth retardation due to reduced level of IGF-1 and, consequently, reduced bone mass. These authors argued that the difference in the results may be due to the different genetic background used in the studies. Similarly a study by Yan and colleagues reported an effect of GM on IGF-1 and consequently on bone growth, the study demonstrated an acute effect of GF colonization with GM obtained from conventional raised mice on reduction of bone mass due to increased bone resorption, whereas the long-term colonization resulted in a net skeletal growth in young animals [59].

Even the studies on mice treated with broad spectrum antibiotics to alter GM bring to different conclusions regarding the effect on bone density, these discrepancies are possibly due to differences in animal age, sex, and protocols applied for antibiotic treatment [59-63].

The majority of the reports suggest that antibiotic treated mice have increased bone density [60, 63, 64] and also best bone mechanical properties [64] than conventional raised mice.

GF mice showed a reduced number of osteoclast, lower level of IL-6, RANKL and TNF $\alpha$ in bone, these cytokines have a well-known pro-inflammatory and pro-osteoclastogenic effect [65, 66], GF mice also displayed alteration of immune system with lower number of CD4+ T cells and no difference of CD8+ T cells, these features are normalized by colonization with GM from conventionally raised mice [65].

Recently elegant studies demonstrated the role of innate immunity in mediating the effect of GM on inflammation and on bone metabolism, in particular the role of toll like receptor 5 (TLR5) [64, 66], Myd88, Nod1 and Nod2 has been studied.

TLR5 is the innate immune receptor for flagellin [67] and mice knock-out (KO) for this receptor develop an altered GM due to deficits in the immune system. TLR5KO mice have an altered hostmicrobe interactions, increased inflammation and metabolic syndrome [68]. It has been demonstrated that metabolic phenotype in these mice depends on GM alteration as TLR5KO mice raised in GF conditions do not develop the metabolic phenotype [69]. Bone phenotype is significantly different in TLR5KO mice as respect to WT, these animals have larger cross-sectional area and moment of inertia with a reduction in whole-bone strength. The effect of antibiotic treatment and disruption of the GM on bone tissue material properties was different between WT and TLR5KO mice, in particular TLR5KO mice display a greater reduction of the whole-bone femoral bending stiffness as respect to WT [64]. These differences may be due to several characteristics of TLR5KO mice: these mice are mildly obese and it is known that obesity influences bone mechanical competence [70]; moreover GM is altered in TLRKO mice that display low microbial diversity, that might, per se, influence bone phenotype; finally immune system is altered in these animals, these could affect GM-immune system-bone interaction.

In order to study the role of innate immunity in mediating the effect of GM on bone health Ohlsson and colleagues [66] evaluated the role of Myd88, NOD1 and NOD2. Myd88 is the main mediator of TLR activity on inflammatory response [71], however Myd88KO mice behave like WT mice when raised in GF environment and display a significant increase in cortical bone mass, this observation demonstrates that the effect of the GM on bone mass is independent of Myd88.

NOD1 and NOD2 bind bacterial peptidoglycan and cooperate to inflammatory response after bacterial recognition in the cytoplasm activating the NFKB pathway. NOD1 detects diaminopimelic acid-type peptidoglycan that is mainly expressed by Gram-negative bacteria [72]. Nod2 detects all types of peptidoglycans found in Gram-positive and Gram-negative bacteria [73].GF mice with deletion of NOD1 or NOD2 do not have increased cortical thickness nor increased expression of 
TNF $\alpha$ and RANKL, thus the effect of GM on the production of this cytokines and, hence, on bone mass is dependent by these molecules.

To investigate the role of GM in bone loss induced by sex steroid deficiency, this condition was induced pharmacologically in GF mice with the GnRH agonists leuprolide by Li and colleagues [74].These authors demonstrated that GM plays an important role in sex steroid deficiency induced osteoporosis: GF mice are protected against osteoporosis and the increase in bone turnover induced by sex steroid deprivation thanks to the lack of increase in TNF, RANKL, and IL-17. The authors also demonstrated that sex steroid depletion augments inflammation in the intestine by increasing gut permeability to bacterial antigens, namely by decreasing the expression of claudin 2, 3, and 15, and of Jam3, which are modulators of intestinal barrier integrity [75, 76].

In humans scarce data support results obtained in mice, recently Wang and colleagues [77] in a very limited cohort suggest that GM component structure and diversity are altered in osteoporosis and osteopenia patients as compared with normal controls, however they do not correlate different GM components with inflammation and immune system, nor with bone turnover.

Relationships between immune system, estrogen deficiency, bone loss and GM are summarized in Fig. 2.

\section{GM and bone health beyond immune system}

It has been suggested that GM composition and manipulation may affect bone health beyond immune system by influencing calcium absorption and the production of gut derived serotonin. A post-hoc analyses on the use of Lactobacillus reuteri demonstrated that the use of this probiotic in healthy subject increases the level of serum $25 \mathrm{OH}$ vitamin $\mathrm{D}$, that influences calcium absorption and benefits bone health. The mechanism through which this probiotic influences vitamin $\mathrm{D}$ level is not clear, however the authors argued that this may be due to a modification in the gut environment that specifically favors vitamin D absorption or to indirect effect on increased hepatic 25hydroxylase activity or 7-dehydrocholesterol concentration due to reduced absorption of dietary and biliary cholesterol [78]. On the other hand the relation between GM and vitamin D may also be inverse as it has been proposed that decreased vitamin D intake is associated with different GM profile $[79,80]$.

Another possible mechanism through which GM benefits bone health is the increase in calcium absorption. It is well known that maintaining a positive calcium balance is important in achieving a good peak of bone mass that protects from the development of osteoporosis in older age [81, 82]. Dietary intake of fibers influences calcium absorption, after being fermented by GM, fibers improve calcium absorption by reduction of gut $\mathrm{pH}$, thus reducing the formation of calcium phosphates and increasing the calcium absorption and by increasing the production of SCFAs as butyrate [83]. The effect of SCFAs may be more complex that the effect on gut $\mathrm{pH}$ and in fact it has been demonstrated that SCFAs increase calcium transport trough signaling pathway modulation [84].As previously said SCFAs influence bone health also trough immune system modulation, hence dietary fibers intake may be responsible for an healthier immune system and reduced inflammation, in fact there is a general consensus recognizing that an adequate dietary fiber intake is associated with lower risk of chronic diseases as cardiovascular diseases [85]. 
Another possible mechanism through which GM influences bone health is mediated by its effect on the production of gut serotonin (5HT). In the recent past a dual effect of serotonin in the regulation of bone mass has been described depending on the site of production of this molecule [86]. In this review we are interested in the role of gut derived 5HT (g5HT), that is influenced by GM, as a bone mass regulator. Enterochromaffin cells of the duodenum are responsible for the synthesis of g5HT that is partially modulated by GM as SCFAs increase the synthesis of g5HT [87, 88]. It has been shown that 5HT interacts with bone cells and, in particular, decreases osteoblast proliferation via activation of 5-HT1B receptors on pre-osteoblasts $[89,90]$.These observations suggest that regulation of g5HT by GM may be a potential therapeutic strategy to improve bone health, indeed, in animal models of ovariectomy induced bone loss, pharmacological inhibition of g5HT synthesis results in prevention of osteoporosis mediated by increased bone formation [91].

However data on the effect of 5HT on bone health are quite controversial, Cui and colleagues [92] showed that mice KO for 5HT receptor 1 have no bone phenotype and that inhibition of this receptor with LP923941, an enantiomer of LP533401 used in a previous study with opposite results [91], decreases circulating 5-HT, but has no effect on bone density. Different results obtained may be explained by different techniques used [93].

Relationships between GM and bone turnover beyond immune system are summarized in Fig. 3 .

\section{GM manipulation and bone health}

GM composition may be manipulated in several ways as the use of broad spectrum antibiotics, change in dietary habits and, more easily, by the use of prebiotics and probiotics, change in GM composition may affect bone health. The majority of experimental data produced in mice demonstrated that modulation of GM by the use of probiotics is able to increase bone mass and to reduce sex steroid associated bone loss [74, 94-96]. Probiotics used were different in different studies, both a single strain or a mixture of strains, the most used were Lactobacilli spp. that were demonstrated to have the higher anti-inflammatory and bone protective effect. McCabe and colleagues suggested that short-term oral administration of the Lactobacillus reuteri enhanced bone density in male, but not in female mice [97], however in estrogen-deficient female mice the administration of this probiotic prevented bone loss [95]. In a further study the authors suggested that $L$. reuteri is active on bone health also in intact females providing the presence of an inflammatory status, the authors speculated that estrogen deficiency is comparable to a mild inflammatory status, thus explaining their previous findings on intact female [98].

Also some data on the use of yogurt that contains different probiotics, but is also a source of calcium and proteins that are fundamental for bone health, have been produced [99]. All these studies showed a protective effect of probiotic yogurt on bone health, moreover it has been demonstrated that dairy products consumption in early life led to a higher peak bone mass [100]. Also in adults older than 60 years consumption of dairy products was associated to increased bone density and lower risk of osteoporosis [101-104]. The use of probiotics has been proposed also as adjuvant treatment in focal bone loss as alveolar erosion in periodontitis, the ability of different Lactobacilli strains in reducing osteoclast number, alveolar erosions and tooth movement in rat and mice has been demonstrated [105-107]. In humans a recent meta-analysis concludes that current 
evidences suggest a possible use of probiotics as adjuvant therapy in gingivitis and periodontitis [108].

In a geriatric population the administration of Lactobacillus helveticus increases serum calcium [109]; in a prospective double-blind, placebo-controlled randomized clinical trial the administration of Lactobacillus casei Shirota in 417 elderly patients with a distal radius fracture accelerates the healing process [110]. Also in osteopenic women the administration of a multispecies probiotic (6 different species) increases markers of bone formation, decreases TNF alpha level, but has no effect on bone density during a 6 months period [111].

Another method to influence GM is the administration of prebiotics, prebiotics are complex carbohydrate and fibers, that influence composition and/or activity of GM in a way that favors host health. To generate beneficial metabolic products GM need substrate availability, prebiotics partially provides these substrates, and can be used to modify the GM components and their metabolites. To be classified as prebiotic a substance should meet these criteria: be resistant to low gastric $\mathrm{pH}$, hydrolysis by mammalian digestive enzymes, and not be absorbable by humans, be fermented by GM and stimulate the growth and activity of gastro intestinal tract [112]. Prebiotic supplementation in animal models favors the proliferation of Bifidobacteria and increases SCFAs production. As regards the effect of prebiotics on bone health some experimental studies showed that they improved calcium absorption and bone density in animal models [113, 114].In humans the supplementation with different probiotics as galacto-oligosaccharide and a mixture of short- and long-chain inulin-type fructans in adolescent girls improved calcium absorption and improved bone density $[115,116]$. Recently the corn-derived non-digestible carbohydrate, soluble corn fiber (SCF), has been evaluated for its ability to increase calcium absorption and improve bone health in humans. In particular SCF administration enhances calcium absorption and its consumption is associated with a favorable change in GM, namely increased presence of Bacteroidetes and Firmicutes known to ferment starch and fiber $[117,118]$. In the study by Whisner and colleagues [117] increase in calcium absorption was positively correlated with bone formation marker, also the changes observed in GM phyla proportion was associated with calcium absorption, Parabacteroides significantly increases with larger SCF doses and negatively correlated with calcium absorption. Firmicutes positively correlated with calcium absorption. The results of this elegant study suggests that the role of GM in calcium absorption is complex and due to different species.

Prebiotic fiber may influence bone metabolism both by the change in composition of GM favoring microbes with higher anti-inflammatory potential and by increasing SCFAs production thus increasing the calcium absorption. It has also been suggested that prebiotics could have direct effect on immune system modulation and an anti-pathogen effects regardless to their effect on GM [119]. However, until now, in human studies on prebiotics only calcium absorption, markers of bone metabolism and bone density were investigated, whereas immune phenotype and inflammation were not.

\section{Conclusions}

GM is becoming one of the new players in the regulation of bone turnover by modulating immune system and controlling inflammation and also by influencing calcium absorption and vitamin D level. 
Dysbiosis may favor bone loss in aged people and after menopause ,manipulation of GM may become a future adjuvant treatment in preventing osteoporosis, osteopenia and other diseases characterized by focal bone loss as periodontitis.

In the last years several data obtained in animal models strongly supported the role of GM in the control of bone turnover, less data have been published in humans, field in which confirmatory studies are needed. In particular large clinical trials are needed to clarify the efficacy of prebiotics and probiotics in favoring bone health during growth, aging and post-menopausal bone loss.

Funding: F. Sassi is supported by a grant from MIUR PRIN 2015.

\section{Compliance with Ethical Standards}

Conflict of interest: the authors declare that they have no conflict of interest.

Ethical Approval: This article does not contain any studies with human participants or animals performed by any of the authors. 


\section{References}

1. Human Microbiome Project Consortium (2012) Structure, function and diversity of the healthy human microbiome. Nature 486:207-214. doi:10.1038/nature11234

2. Schroeder BO, Bäckhed F (2016) Signals from the gut microbiota to distant organs in physiology and disease. Nat Med 22:1079-1089. doi:10.1038/nm.4185

3. Le Chatelier E, Nielsen T, Qin J et al (2013) Richness of human gut microbiome correlates with metabolic markers. Nature 500:541-546. doi:10.1038/nature12506

4. Statovci D, Aguilera M, MacSharry J, Melgar (2017) The impact of western diet and nutrients on the microbiota and immune response at mucosal interfaces. Front Immunol 8:838. doi:10.3389/fimmu.2017.00838

5. Sokol H, Jegou S, McQuitty C et al (2017) Specificities of the intestinal microbiota in patients with inflammatory bowel disease and Clostridium difficile infection. Gut Microbes 8:0. doi:10.1080/19490976.2017.1361092

6. Crovesy L, Ostrowski M, Ferreira DMTP, Rosado EL, Soares-Mota M (2017) Effect of Lactobacillus on body weight and body fat in overweight subjects: a systematic review of randomized controlled clinical trials. Int J Obes, doi: 10.1038/ijo.2017.161, July 10, 2004

7. Arumugam M, Raes J, Pelletier E et al (2011) Enterotypes of the human gut microbiome. Nature 473:174-180. doi:10.1038/nature09944

8. Peterson CT, Sharma V, Elmén L, Peterson SN (2015) Immune homeostasis, dysbiosis and therapeutic modulation of the gut microbiota. Clin Exp Immunol 179:363-377. doi:10.1111/cei.12474

9. Belkaid Y, Hand TW (2014) Role of the microbiota in immunity and inflammation. Cell 157:121-141. doi:10.1016/j.cell.2014.03.011

10. Wu HJ, Wu E (2012) The role of gut microbiota in immune homeostasis and autoimmunity. Gut Microbes 3:4-14. doi:10.4161/gmic.19320

11. Bauer H, Horowitz RE, Levenson SM, Popper H (1963) The response of the lymphatic tissue to the microbial flora. Studies on germfree mice. Am J Pathol 42:471-483

12. Hamada H, Hiroi T, Nishiyama Y et al (2002) Identification of multiple isolated lymphoid follicles on the antimesenteric wall of the mouse small intestine. J Immunol 168:57-64

13. Macpherson AJ,Hunziker L, McCoy K, Lamarre A (2001) IgA responses in the intestinal mucosa against pathogenic and non-pathogenic microorganisms. Microbes Infect 3:10211035 
14. Mazmanian SK, Liu CH, Tzianabos AO, Kasper DL (2005) An immunomodulatory molecule of symbiotic bacteria directs maturation of the host immune system. Cell 122:107118. doi:10.1016/j.cell.2005.05.007

15. Smith K, McCoy KD, Macpherson AJ (2007) Use of axenic animals in studying the adaptation of mammals to their commensal intestinal microbiota. Semin Immunol 19:59-69. doi:10.1016/j.smim.2006.10.002

16. TalhamGL, Jiang HQ, Bos NA, Cebra JJ (1999) Segmented filamentous bacteria are potent stimuli of a physiologically normal state of the murine gut mucosal immune system. Infect Immun 67:1992-2000

17. Bouskra D, Brézillon C, Bérard M et al (2008) Lymphoid tissue genesis induced by commensals through NOD1 regulates intestinal homeostasis. Nature 456:507-510. doi:10.1038/nature07450

18. Ohnmacht C, Marques R, Presley L, Sawa S, Lochner M, Eberl G (2011) Intestinal microbiota, evolution of the immune system and the bad reputation of pro-inflammatory immunity. Cell Microbiol 13:653-659. doi:10.1111/j.1462-5822.2011.01577.x

19. Wu HJ, Ivanov II, Darce J et al (2010) Gut-residing segmented filamentousbacteria drive autoimmune arthritis via T helper 17 cells. Immunity 32:815-827.

doi:10.1016/j.immuni.2010.06.001

20. Scher JU, Sczesnak A, Longman RS et al (2013) Expansion of intestinal Prevotella copri correlates with enhanced susceptibility to arthritis. eLife 2:e01202. doi:10.7554/eLife.01202

21. Maeda Y, Kurakawa T, Umemoto E et al (2016) Dysbiosis contributes to arthritis development via activation of autoreactive T cells in the intestine. Arthritis Rheumatol 68:2646-2661. doi:10.1002/art.39783

22. Marietta EV, Murray JA, Luckey DH et al (2016) Suppression of inflammatory arthritis by human gut-derived Prevotella histicola in humanized mice. Arthritis Rheumatol 68:28782888. doi:10.1002/art.39785

23. Atarashi K, Tanoue T, Shima $\mathrm{T}$ et al (2011) Induction of colonic regulatory $\mathrm{T}$ cells by indigenous Clostridium species. Science 331:337-341. doi:10.1126/science.1198469

24. Gaboriau-Routhiau V, Rakotobe S, Lécuyer E et al (2009) The key role of segmented filamentous bacteria in the coordinated maturation of gut helper $\mathrm{T}$ cell responses. Immunity 31:677-689. doi:10.1016/j.immuni.2009.08.020

25. Wu X, He B, Liu J et al (2016) Molecular insight into gut microbiota and rheumatoid arthritis. Int J MolSci 17:431. doi:10.3390/ijms17030431 
26. Lee N, Kim WU (2017) Microbiota in T-cell homeostasis and inflammatory diseases. Exp Mol Med 49:e340. doi:10.1038/emm.2017.36

27. Ferreira CM, Vieira AT, Vinolo MA, Oliveira FA, Curi R, Martins Fdos S (2014) The central role of the gut microbiota in chronic inflammatory diseases. J Immunol Res 2014:689492. doi:10.1155/2014/689492

28. Furusawa Y, Obata Y, Fukuda S et al (2013) Commensal microbe-derived butyrate induces the differentiation of colonic regulatory T cells. Nature 504:446-450. doi:10.1038/nature12721

29. Smith PM, Howitt MR, Panikov N et al (2013) The microbial metabolites, short-chain fatty acids, regulate colonic Treg cell homeostasis. Science 341:569-573. doi:10.1126/science. 1241165

30. Brown AJ, Goldsworthy SM, Barnes AA et al (2003) The Orphan G protein-coupled receptors GPR41 and GPR43 are activated by propionate and other short chain carboxylic acids. J Biol Chem 278:11312-11319. doi:10.1074/jbc.M211609200

31. Le Poul E, Loison C, Struyf S et al (2003) Functional characterization of human receptors for short chain fatty acids and their role in polymorphonuclear cell activation. J Biol Chem 278:25481-25489. doi:10.1074/jbc.M301403200

32. Nilsson NE, Kotarsky K, Owman C, Olde B (2003) Identification of a free fatty acid receptor, FFA2R, expressed on leukocytes and activated by short-chain fatty acids. Biochem Biophys Res Commun 303:1047-1052

33. Thangaraju M, Cresci GA, Liu K et al (2009) GPR109A is a G-protein-coupled receptor for the bacterial fermentation product butyrate and functions as a tumor suppressor in colon. Cancer Res 69:2826-2832. doi:10.1158/0008-5472.CAN-08-4466

34. Singh N, Gurav A, Sivaprakasam S et al (2014) Activation of Gpr109a, receptor for niacin and the commensal metabolite butyrate, suppresses colonic inflammation and carcinogenesis. Immunity 40:128-139. doi:10.1016/j.immuni.2013.12.007

35. Arpaia N, Campbell C, Fan X et al (2013) Metabolites produced by commensal bacteria promote peripheral regulatory T-cell generation. Nature 504:451-455.

doi:10.1038/nature12726

36. Davie JR (2003) Inhibition of histone deacetylase activity by butyrate. J Nutr 133:2485S2493S 
37. Thangaraju M, Carswell KN, Prasad PD, Ganapathy V (2009). Colon cancer cells maintain low levels of pyruvate to avoid cell death caused by inhibition of HDAC1/HDAC3. Biochem J 417:379-389. doi:10.1042/BJ20081132

38. Sanford JA, Zhang LJ, Williams MR, Gangoiti JA, Huang CM, Gallo RL (2016) Inhibition of HDAC8 and HDAC9 by microbial short-chain fatty acids breaks immune tolerance of the epidermis to TLR ligands. Sci Immunol 1:pii:eaah4609. doi:10.1126/sciimmunol.aah4609

39. Huang J, Wang L, Dahiya S et al (2017) Histone/protein deacetylase 11 targeting promotes Foxp3+ Treg function. Sci Rep 7:8626. doi:10.1038/s41598-017-09211-3

40. Park J, Kim M, Kang SG et al (2015) Short-chain fatty acids induce both effector and regulatory $\mathrm{T}$ cells by suppression of histone deacetylases and regulation of the mTOR-S6K pathway. Mucosal Immunol 8:80-93. doi:10.1038/mi.2014.44

41. Liu L, Li L, Min J et al (2012) Butyrate interferes with the differentiation and function of human monocyte-derived dendritic cells. Cell Immunol277:6673. doi:10.1016/j.cellimm.2012.05.011

42. Millard AL, Mertes PM, Ittelet D, Villard F, Jeannesson P, Bernard J (2002) Butyrate affects differentiation, maturation and function of human monocyte-derived dendritic cells and macrophages. Clin Exp Immunol 130:245-255

43. Singh N, Thangaraju M, Prasad PD et al (2010) Blockade of dendritic cell development by bacterial fermentation products butyrate and propionate through a transporter (Slc5a8)dependent inhibition of histone deacetylases. J Biol Chem 285:27601-27608. doi:10.1074/jbc.M110.102947

44. Wang B, Morinobu A, Horiuchi M, Liu J, Kumagai S (2008) Butyrate inhibits functional differentiation of human monocyte-derived dendritic cells. Cell Immunol 253:54-58. doi:10.1016/j.cellimm.2008.04.016

45. Trompette A, Gollwitzer ES, Yadava K et al (2014) Gut microbiota metabolism of dietary fiber influences allergic airway disease and hematopoiesis. Nat Med 20:159-166. doi:10.1038/nm.3444

46. Postler TS, Ghosh S (2017) Understanding the holobiont: how microbial metabolites affect human health and shape the immune system. Cell Metab 26:110-130.

doi:10.1016/j.cmet.2017.05.008

47. Round JL, Lee SM, Li J et al (2011) The Toll-like receptor 2 pathway establishes colonization by a commensal of the human microbiota. Science 332:974-977. doi:10.1126/science.1206095 
48. Ivanov II, Atarashi K, Manel N et al (2009) Induction of intestinal Th17 cells by segmented filamentous bacteria. Cell 139:485-498. doi:10.1016/j.cell.2009.09.033

49. Kim M, Qie Y, Park J, Kim CH (2016) Gut microbial metabolites fuel host antibody responses. Cell Host Microbe 20:202-214. doi:10.1016/j.chom.2016.07.001

50. Longman RS, Yang Y, Diehl GE, Kim SV, Littman DR (2013) Microbiota: host interactions in mucosal homeostasis and systemic autoimmunity. Cold Spring Harb Symp Quant Biol 78:193-201. doi:10.1101/sqb.2013.78.020081

51. Lane ER, Zisman TL, Suskind DL (2017) The microbiota in inflammatory bowel disease: current and therapeutic insights. J Inflamm Res 10:63-73. doi:10.2147/JIR.S116088

52. Maeda Y, Takeda K (2017) Role of gut microbiota in rheumatoid arthritis. J Clin Med 6.pii:E60. doi:10.3390/jcm6060060

53. Wen L, Ley RE, Volchkov PY et al (2008) Innate immunity and intestinal microbiota in the development of Type 1 diabetes. Nature 455:1109-1113. doi:10.1038/nature07336

54. Shukla SD, Budden KF, Neal R, Hansbro PM (2017) Microbiome effects on immunity, health and disease in the lung. Clin Transl Immunology 6:e133. doi:10.1038/cti.2017.6

55. Hernlund E, Svedbom A, Ivergård M et al (2013) Osteoporosis in the European Union: medical management, epidemiology and economic burden. A report prepared in collaboration with the International Osteoporosis Foundation (IOF) and the European Federation of Pharmaceutical Industry Associations (EFPIA). Arch Osteoporos 8:136. doi:10.1007/s11657-013-0136-1

56. Mori G, D'Amelio P, Faccio R, Brunetti G (2015) Bone-immune cellcrosstalk: bone diseases. J Immunol Res 2015:108451. doi:10.1155/2015/108451

57. D'Amelio P, Grimaldi A, Di Bella S et al (2008) Estrogen deficiency increases osteoclastogenesis up-regulating $\mathrm{T}$ cells activity: a key mechanism in osteoporosis. Bone 43:92-100. doi:10.1016/j.bone.2008.02.017

58. Schwarzer M, Makki K, Storelli G et al (2016) Lactobacillus plantarum strain maintains growth of infant mice during chronic undernutrition. Science 351:854-857. doi:10.1126/science.aad8588

59. Yan J, Herzog JW, Tsang K et al (2016) Gut microbiota induce IGF-1 and promote bone formation and growth. Proc Natl Acad Sci U S A 113:E7554-E7563. doi:10.1073/pnas.1607235113

60. Cho I, Yamanishi S, Cox L et al (2012) Antibiotics in early life alter the murine colonic microbiome and adiposity. Nature 488:621-626. doi:10.1038/nature11400 
61. Cox LM, Yamanishi S, Sohn J et al (2014) Altering the intestinal microbiota during a critical developmental window has lasting metabolic consequences. Cell 158:705-721. doi:10.1016/j.cell.2014.05.052

62. Nobel YR, Cox LM, Kirigin FF et al (2015) Metabolic and metagenomic outcomes from early-life pulsed antibiotic treatment. Nat Commun 6:7486. doi:10.1038/ncomms8486

63. Pytlik M, Folwarczna J, Janiec W (2004) Effects of doxycycline on mechanical properties of bones in rats with ovariectomy-induced osteopenia. Calcif Tissue Int 75:225-230. doi:10.1007/s00223-004-0097-x

64. Guss JD, Horsfield MW, Fontenele FF et al (2017) Alterations to the gut microbiome impair bone strength and tissue material properties. J Bone Miner Res 32:1343-1353. doi:10.1002/jbmr.3114

65. Sjögren K, Engdahl C, Henning P et al (2012) The gut microbiota regulates bone mass in mice. J Bone Miner Res 27:1357-1367. doi:10.1002/jbmr.1588

66. Ohlsson C, Nigro G, Boneca IG, Bäckhed F, Sansonetti P, Sjögren K (2017) Regulation of bone mass by the gut microbiota is dependent on NOD1 and NOD2 signaling. Cell Immunol 317:55-58. doi:10.1016/j.cellimm.2017.05.003

67. Hayashi F, Smith KD, Ozinsky A et al (2001) The innate immune response to bacterial flagellin is mediated by Toll-like receptor 5. Nature 410:1099-1103. doi:10.1038/35074106

68. Cullender TC, Chassaing B, Janzon A et al (2013) Innate and adaptive immunity interact to quench microbiome flagellar motility in the gut. Cell Host Microbe 14:571-581. doi:10.1016/j.chom.2013.10.009

69. Vijay-Kumar M, Aitken JD, Carvalho FA et al (2010) Metabolic syndrome and altered gut microbiota in mice lacking Toll-like receptor 5. Science 328:228-231.

doi:10.1126/science. 1179721

70. Ionova-Martin SS, Wade JM, Tang S et al (2011) Changes in cortical bone response to highfat diet from adolescence to adulthood in mice. Osteoporos Int 22:2283-2293.

doi:10.1007/s00198-010-1432-x

71. Kufer TA, Sansonetti PJ (2007) Sensing of bacteria: NOD a lonely job. Curr Opin Microbiol 10:62-69. doi:10.1016/j.mib.2006.11.003

72. Clarke TB, Davis KM, Lysenko ES, Zhou AY, Yu Y, Weiser JN (2010) Recognition of peptidoglycan from the microbiota by Nod1 enhances systemic innate immunity. Nat Med 16:228-231. doi:10.1038/nm.2087 
73. Nigro G, Rossi R, Commere PH, Jay P, Sansonetti PJ (2014) The cytosolic bacterial peptidoglycan sensor Nod2 affords stem cell protection and links microbes to gut epithelial regeneration. Cell Host Microbe 15:792-798. doi:10.1016/j.chom.2014.05.003

74. Li JY, Chassaing B, Tyagi AM et al (2016) Sex steroid deficiency-associated bone loss is microbiota dependent and prevented by probiotics. J Clin Invest 126:2049-2063. doi:10.1172/JCI86062

75. Zeissig S, Bürgel N, Günzel D et al (2007) Changes in expression and distribution of claudin 2, 5 and 8 lead to discontinuous tight junctions and barrier dysfunction in active Crohn's disease. Gut 56:61-72. doi:10.1136/gut.2006.094375

76. Grootjans J, Thuijls G, Verdam F, Derikx JP, Lenaerts K, Buurman WA (2010) Noninvasive assessment of barrier integrity and function of the human gut. World $\mathrm{J}$ GastrointestSurg 2:61-69. doi:10.4240/wjgs.v2.i3.61

77. Wang J, Wang Y, Gao Wet al (2017) Diversity analysis of gut microbiota in osteoporosis and osteopenia patients. Peer J 5:e3450. doi:10.7717/peerj.3450

78. Jones ML, Martoni CJ, Prakash S (2013) Oral supplementation with probiotic L. reuteri NCIMB 30242 increases mean circulating 25-hydroxyvitamin D: a post hoc analysis of a randomized controlled trial. J Clin Endocrinol Metab 98:944-2951. doi:10.1210/jc.20124262

79. Yoon SS, Sun J (2011) Probiotics, nuclear receptor signaling, and anti-inflammatory pathways. Gastroenterol Res Pract 2011:971938. doi:10.1155/2011/971938

80. Ly NP, Litonjua A, Gold DR, Celedón JC (2011) Gut microbiota, probiotics, and vitamin D: interrelated exposures influencing allergy, asthma, and obesity? J Allergy Clin Immunol 127:1087-1094.doi:10.1016/j.jaci.2011.02.015

81. Weaver CM, Gordon CM, Janz KF et al (2016) The National Osteoporosis Foundation's position statement on peak bone mass development and lifestyle factors: a systematic review and implementation recommendations. Osteoporos Int 27:1281-1386. doi:10.1007/s00198015-3440-3

82. D'Amelio P, Tamone C, Pluviano F, Di Stefano M, Isaia G (2005) Effects of lifestyle and risk factors on bone mineral density in a cohort of Italian women: suggestion for a new decision rule. Calcif Tissue Int 77:72-78. doi:10.1007/s00223-004-0253-3

83. Wallace TC, Marzorati M, Spence L, Weaver CM, Williamson PS (2017) New frontiers in fibers: innovative and emerging research on the gut microbiome and bone health. J Am Coll Nutr 36:218-222. doi:10.1080/07315724.2016.1257961 
84. Weaver CM (2015) Diet, gut microbiome, and bone health. Curr Osteoporos Rep 13:125130. doi:10.1007/s11914-015-0257-0

85. U.S. Department of Health and Human Services and U.S. Departmentof Agriculture (2015) 2015-2020 Dietary Guidelines for Americans. 8th edn. Washington, DC. http://health.gov/dietaryguidelines/2015/guidelines

86. D'Amelio P, Panico A, Spertino E, Isaia GC (2012) Energy metabolism and the skeleton: reciprocal interplay. World J Orthop 3:190-198. doi:10.5312/wjo.v3.i11.190

87. Reigstad CS, Salmonson CE, Rainey JF $3^{\text {rd }}$ et al (2015) Gut microbes promote colonic serotonin production through an effect of short-chain fatty acids on enterochromaffin cells. FASEB J 29:1395-1403. doi:10.1096/fj.14-259598

88. Yano JM, Yu K, Donaldson GP et al (2015) Indigenous bacteria from the gut microbiota regulate host serotonin biosynthesis. Cell 161:264-276. doi:10.1016/j.cell.2015.02.047

89. Yadav VK, Ryu JH, Suda N et al (2008) Lrp5 controls bone formation by inhibiting serotonin synthesis in the duodenum. Cell 135:825-837. doi:10.1016/j.cell.2008.09.059

90. Kode A, Mosialou I, Silva BC et al (2012) FOXO1 orchestrates the bone-suppressing function of gut-derived serotonin. J Clin Invest 122:3490-3503. doi:10.1172/JCI64906

91. Yadav VK, Balaji S, Suresh PS et al (2010) Pharmacological inhibition of gut-derived serotonin synthesis is a potential bone anabolic treatment for osteoporosis. Nat Med 16:308312. doi: $10.1038 / \mathrm{nm} .2098$

92. Cui Y1, Niziolek PJ, MacDonald BT et al (2011) Lrp5 functions in bone to regulate bone mass. Nat Med 17:684-691. doi:10.1038/nm.2388

93. De Vernejoul MC, Collet C, Chabbi-Achengli Y (2012) Serotonin: good or bad for bone. Bonekey Rep 1:120. doi: 10.1038/bonekey.2012.120

94. Ohlsson C, Engdahl C, Fåk F et al (2014) Probiotics protect mice from ovariectomy-induced cortical bone loss. PLoS One 9:e92368. doi:10.1371/journal.pone.0092368

95. Britton RA, Irwin R, Quach D et al (2014) Probiotic L. reuteri treatment prevents bone loss in a menopausal ovariectomized mouse model. J Cell Physiol 229:1822-1830.

doi:10.1002/jcp.24636

96. Parvaneh K, Ebrahimi M, Sabran MR et al (2015) Probiotics (Bifidobacterium longum) increase bone mass density and upregulate SPARC and BMP-2 genes in rats with bone loss resulting from ovariectomy. Biomed Res Int 2015:897639. doi:10.1155/2015/897639 
97. McCabe LR, Irwin R, Schaefer L, Britton RA (2013) Probiotic use decreases intestinal inflammation and increases bone density in healthy male but not female mice. J Cell Physiol 228:1793-1798. doi:10.1002/jcp.24340

98. Collins FL, Irwin R, Bierhalter H et al (2016) Lactobacillus reuteri 6475 increases bone density in intact females only under an inflammatory setting. PLoS One 11:e0153180. doi:10.1371/journal.pone.0153180

99. Rozenberg S, Body JJ, Bruyère Oet al (2016) Effects of dairy products consumption on health: benefits and beliefs--a commentary from the belgian bone club and the european society for clinical and economic aspects of osteoporosis, osteoarthritis and musculoskeletal diseases. Calcif Tissue Int 98:1-17. doi:10.1007/s00223-015-0062-X

100. Matkovic V, Landoll JD, Badenhop-Stevens NE et al (2004) Nutrition influences skeletal development from childhood to adulthood: a study of hip, spine, and forearm in adolescent females. J Nutr 134:701s-705s

101. Langsetmo L, Barr SI, Berger C et al (2015) Associations of protein intake and protein source with bone mineral density and fracture risk: a population-based cohort study. J Nutr Health Aging 19:861-868. doi:10.1007/s12603-015-0544-6

102. Durosier-Izart C, Biver E, Merminod F et al (2017) Peripheral skeleton bone strength is positively correlated with total and dairy protein intakes in healthy postmenopausal women. Am J Clin Nutr 105:513-525. doi:10.3945/ajcn.116.134676

103. Radavelli-Bagatini S, Zhu K, Lewis JR, Prince RL (2014) Dairy food intake, peripheral bone structure, and muscle mass in elderly ambulatory women. J Bone Miner Res 29:16911700. doi:10.1002/jbmr.2181

104. Laird E, Molloy AM, McNulty H et al (2017) Greater yogurt consumption is associated with increased bone mineral density and physical function in older adults. Osteoporos Int 28:2409-2419. doi:10.1007/s00198-017-4049-5

105. Pazzini CA, Pereira LJ, da Silva TA et al (2017) Probiotic consumption decreases the number of osteoclasts during orthodontic movement in mice. Arch Oral Biol 79:30-34. doi:10.1016/j.archoralbio.2017.02.017

106. Ricoldi MST, Furlaneto FAC, Oliveira LFF et al (2017) Effects of the probiotic Bifidobacterium animalis subsp. lactis on the non-surgical treatment of periodontitis. A histomorphometric, microtomographic and immunohistochemical study in rats. PLoS One 12:e0179946. doi:10.1371/journal.pone.0179946

107. Kobayashi R, Kobayashi T, Sakai F, Hosoya T, Yamamoto M, Kurita-Ochiai T (2017) Oral administration of Lactobacillus gasseri SBT2055 is effective in preventing 
Porphyromonasgingivalis-accelerated periodontal disease. Sci Rep 7:545.

doi:10.1038/s41598-017-00623-9

108. Gruner D, Paris S, Schwendicke F (2016) Probiotics for managing caries and periodontitis: systematic review and meta-analysis. J Dent 48:16-25. doi:10.1016/j.jdent.2016.03.002

109. Gohel MK, Prajapati JB, Mudgal SV et al (2016) Effect of probiotic dietary intervention on calcium and haematological parameters in geriatrics. J Clin Diagn Res 10:LC05-LC09. doi:10.7860/JCDR/2016/18877.7627

110. Lei M, Hua LM, Wang DW (2016) The effect of probiotic treatment on elderly patients with distal radius fracture: a prospective double-blind, placebo-controlled randomised clinical trial. Benef Microbes 7:631-637. doi:10.3920/BM2016.0067

111. Jafarnejad S, Djafarian K, Fazeli MR, Yekaninejad MS, Rostamian A, Keshavarz SA (2017) Effects of a multispecies probiotic supplement on bone health in osteopenic postmenopausal women: a randomized, double-blind, controlled trial. J Am Coll Nutr 19:110. doi:10.1080/07315724.2017.1318724

112. Roberfroid M (2007) Prebiotics: the concept revisited. J Nutr 137:830S-837S

113. Weaver CM, Martin BR, Nakatsu CH et al (2011) Galactooligosaccharides improve mineral absorption and bone properties in growing rats through gut fermentation. J Agric Food Chem 59:6501-6510. doi:10.1021/jf2009777

114. Scholz-Ahrens KE, Schaafsma G, van den Heuvel EG, Schrezenmeir J (2001) Effects of prebiotics on mineral metabolism. Am J ClinNutr 73:459S-464S

115. Whisner CM, Martin BR, Schoterman MH et al (2013) Galacto-oligosaccharides increase calcium absorption and gut Bifidobacteria in young girls: a double-blind cross-over trial. $\mathrm{Br}$ J Nutr 110:1292-1303. doi:10.1017/S000711451300055X

116. Abrams SA, Griffin IJ, Hawthorne KM et al (2005) A combination of prebiotic short- and long-chain inulin-type fructans enhances calcium absorption and bone mineralization in young adolescents. Am J Clin Nutr 82:471-476

117. Whisner CM, Martin BR, Nakatsu CH et al (2016) Soluble corn fiber increases calcium absorption associated with shifts in the gut microbiome: a randomized dose-response trial in free-living pubertal females. J Nutr 146:1298-1306. doi:10.3945/jn.115.227256

118. Whisner CM, Martin BR, Nakatsu CH et al (2014) Soluble maize fibre affects short-term calcium absorption in adolescent boys and girls: a randomised controlled trial using dual stable isotopic tracers. Br J Nutr 112:446-456. doi:10.1017/S0007114514000981 
119. Bindels LB, Delzenne NM, Cani PD, Walter J (2015) Towards a more comprehensive concept for prebiotics. Nat Rev Gastroenterol Hepatol 12:303-310. doi:10.1038/nrgastro.2015.47 


\section{Figure legends}

Fig. 1 The cartoon summarizes how gut microbiota influences enteral barrier integrity and immune system through the production of several metabolites

Abbreviations used: enteral cells (EC), goblet cells (GC), antigen presenting cells (APC); T regulatory cells (Treg); T helper-1 (Th1), Thelper-17 cells (Th17), Short Chain Fatty Acid (SCFAs)

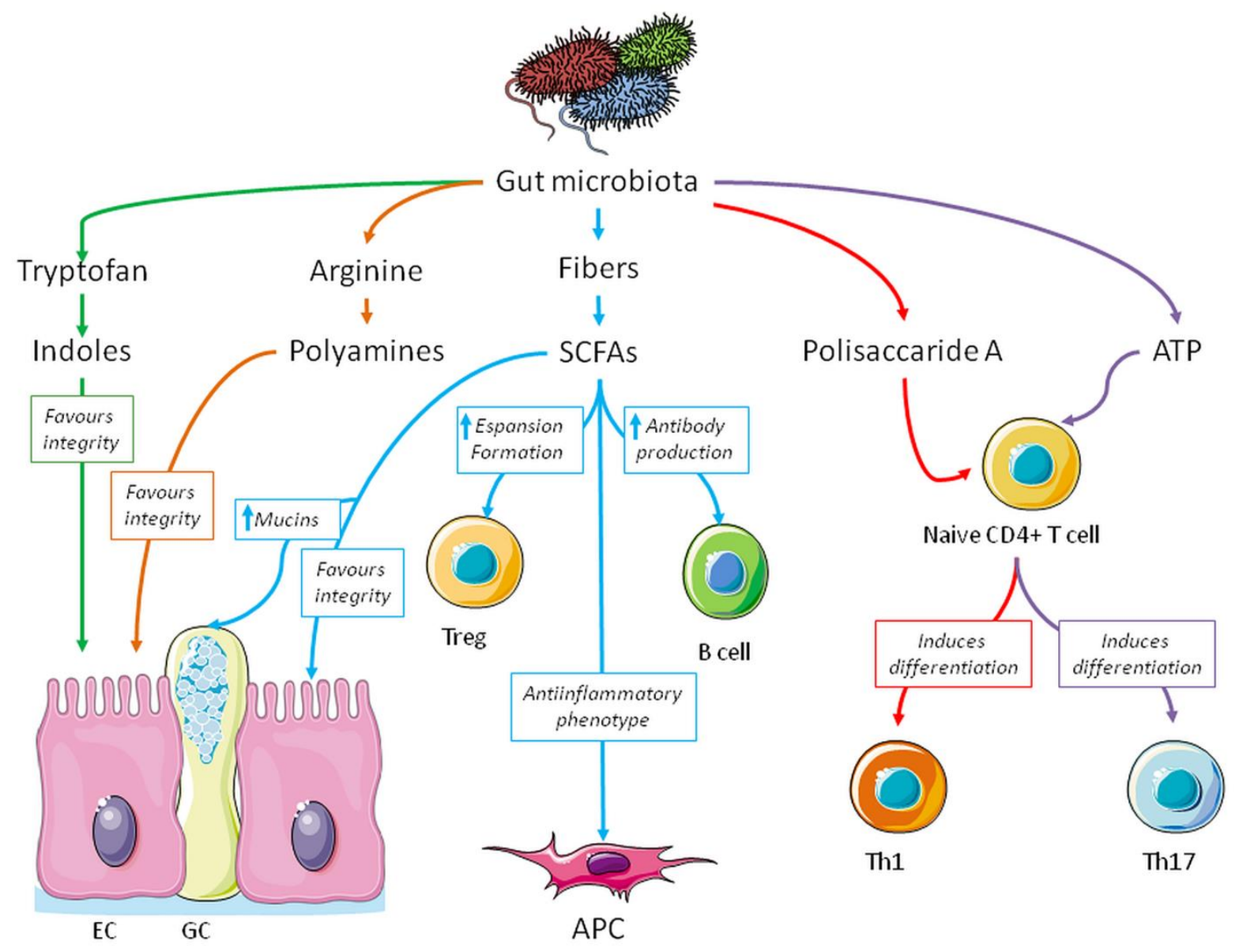


Fig. 2 The cartoon summarizes the complex relationships between immune system, estrogen deficiency-bone loss and gut microbiota: enteral barrier integrity, cytokine production, immune and bone cells are involved

Abbreviations used: gut microbiota (GM) enteral cells (EC), antigen presenting cells (APC); T regulatory cells (Treg); T helper-1 (Th1), Thelper-17 cells (Th17), osteoblasts (OBs), osteoclasts (OCs)

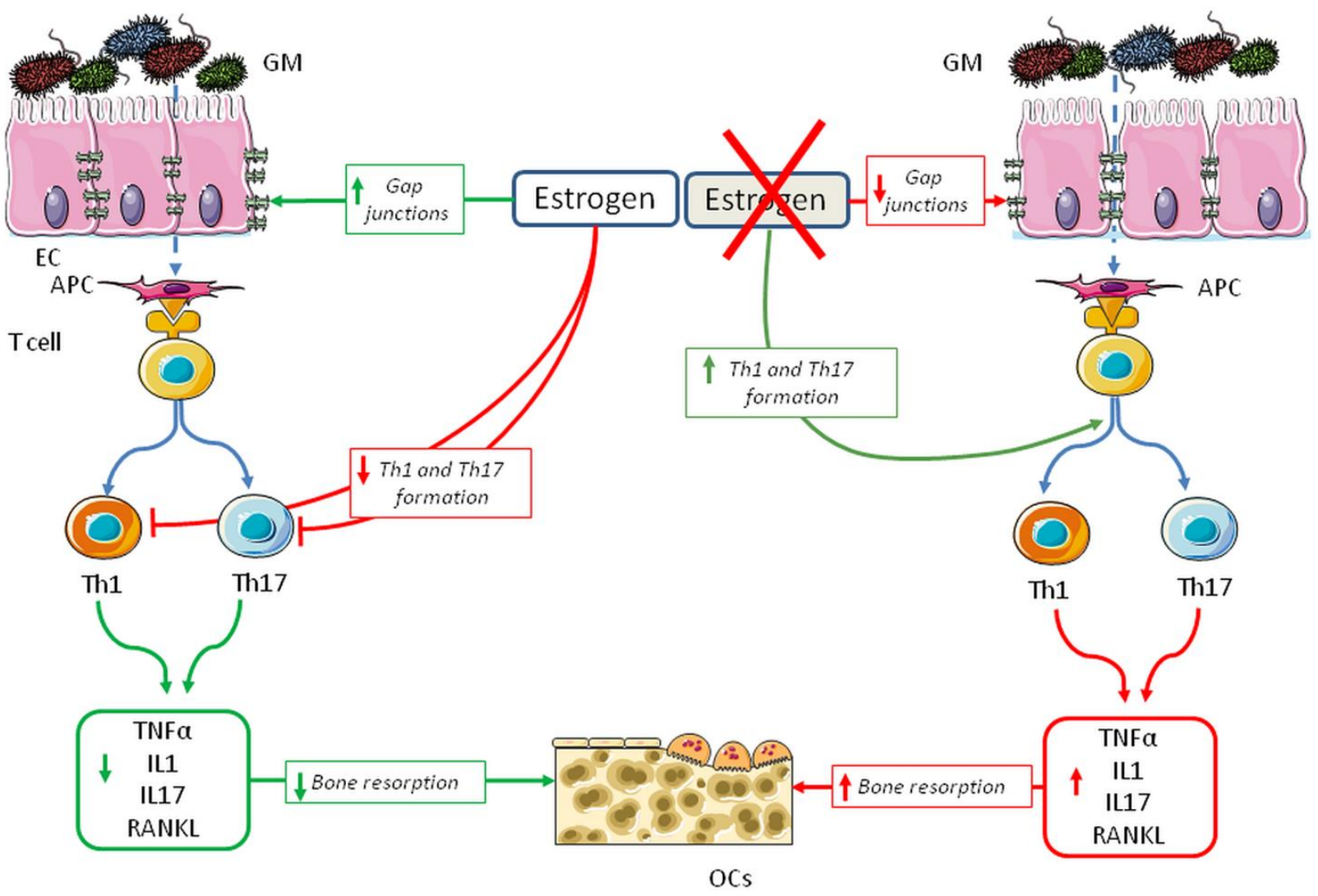


Fig. 3 The cartoon summarizes the link between gut microbiota and bone turnover beyond immune system

Abbreviations used: gut microbiota (GM), enteral cells (EC), enterochromaffin cells (ECC), osteoblasts (OBs)

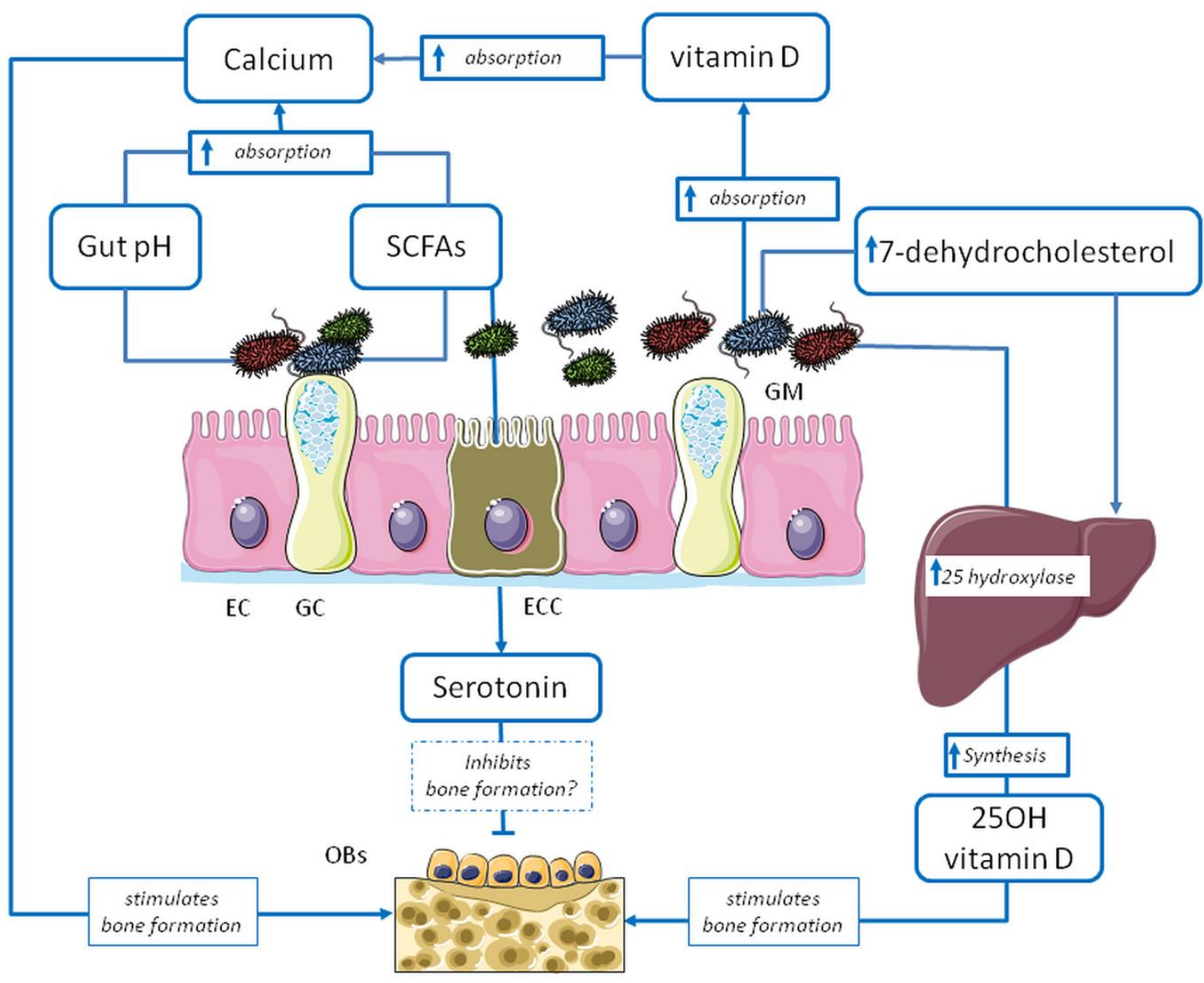

